\title{
Current Methods of Pharmacologic Stress Testing and the Potential Advantages of New Agents*
}

\author{
Elias H. Botvinick
}

Divisions of Cardiology and Nuclear Medicine, Departments of Medicine and Radiology, University of California San Francisco, San Francisco, California

\begin{abstract}
This article presents the exciting advances made and ongoing in the area of pharmacologic cardiac stress testing. In particular, new $\mathrm{A}_{2 \mathrm{~A}}$-specific receptor agonists work like adenosine but promise the delivery of uncomplicated vasodilator stress testing or the diagnosis and prognosis of coronary disease. These agents, although not perfect, do likely present a level of protection against the complications of bronchospasm and heart block. Phase III studies have shown that these agents promise a reduced symptom intensity and greater patient tolerance. One of these agents, regadenoson, is now Food and Drug Administration approved and will be delivered as the same single-dose bolus in all patients, regardless of weight, greatly simplifying the method and increasing its acceptability. Most widely applied with myocardial perfusion SPECT, these agents will find application with PET myocardial perfusion studies and likely MRI studies. Because of their effect on coronary supply rather than demand, they will not be applied with stress echocardiography. Before considering these agents, we will consider the principles and methods of stress testing, and particularly pharmacologic stress testing. The learning objectives of this article are to familiarize the reader with the methods and choices in stress testing for coronary disease diagnosis and prognosis, to present the advantages and disadvantages of pharmacologic stress testing, to review current pharmacologic stress-testing methods and their specific combination with imaging methods, to present the chemistry and effects of the new $\mathrm{A}_{2 \mathrm{a}}$-specific receptor agonists and their advantages compared with existing nonspecific agents, and to help the reader better understand the clinical role of the $\mathrm{A}_{2 \mathrm{a}}$-specific receptor agonists and their application.
\end{abstract}

Key Words: cardiology (clinical); SPECT/CT; MRI; coronary artery disease; perfusion scintigraphy; pharmacologic stress; stress testing; vasodilator

J Nucl Med Technol 2009; 37:14-25

DOI: $10.2967 /$ jnmt.108.057802

$\mathbf{T}$ he objective of all forms of stress testing in coronary artery disease (CAD) is to assess the extent and adequacy of

Received Sep. 9, 2008; revision accepted Dec. 24, 2008.

For correspondence or reprints contact: Elias H. Botvinick, Box 0214, University of California San Francisco, 505 Parnassus Ave., Room M-308, San Francisco, CA 94143-0214.

E-mail: botvinicke@medicine.ucsf.edu

*NOTE: FOR CE CREDIT, YOU CAN ACCESS THIS ACTIVITY THROUGH THE SNM WEB SITE (http://www.snm.org/ce_online) THROUGH March 2011.

COPYRIGHT (C) 2009 by the Society of Nuclear Medicine, Inc. the hyperemic response, testing the ability of the coronary circulation to augment flow (the coronary flow reserve [CFR]) (1). Stress testing elicits and evaluates the ischemic indicators or endpoints, symptoms or signs that may relate to a coronary supply or demand imbalance characteristic of CAD. These symptoms or signs occur in a sequence related to the extent and duration of induced ischemia. This ischemic cascade (2) includes abnormalities of perfusion, the first and precipitating event, followed by myocardial stiffening, then wall motion abnormalities, electrocardiographic ST segment changes, and chest pain. Although MRI methods now image the coronary hyperemic response, MRI has not been widely evaluated and is not widely available. The stress perfusion endpoint, induced flow heterogeneity (which is widely available currently only scintigraphically using myocardial perfusion imaging [MPI]), seems most advantageous compared with the functional ischemic endpoint, induced wall motion abnormalities (available with blood-pool imaging, echocardiography, or MRI) (Table 1).

The hyperemic response, assessed with perfusion or functional endpoints, may be tested with dynamic exercise or dobutamine. The coronary dilators dipyridamole and adenosine induce flow heterogeneity but do not generally produce ischemia in the presence of a significant, flowlimiting coronary stenosis. Thus, they require combination with an imaging method to measure the flow response, most commonly MPI. Clinically, exercise testing, when appropriate, is always preferred to pharmacologic stress testing. Exercise testing provides information regarding patient performance and permits an evaluation of exercise-related symptoms and of the relationship between activities and symptoms. Adenosine is the naturally occurring ligand of 4 distinct subtypes $\left(A_{1}, A_{2 a}, A_{2 b}\right.$, and $\left.A_{3}\right)$ of cell membrane $G$ protein-coupled receptors. Because of the difference in receptors, intermediates, and related pathways to coronary dilation, the vasodilator response to these interventions, exercise and pharmacologic stress, may differ (4).

\section{EXERCISE AND PHARMACOLOGIC STRESS TESTING FOR CAD EVALUATION}

Dynamic exercise may be viewed as an indirect test of the CFR. This type of exercise may be performed on a treadmill or bicycle and (primarily through its effect on heart rate, 
TABLE 1

Characteristics of Stress Ischemic Endpoints

\begin{tabular}{ll}
\hline \multicolumn{1}{c}{ Perfusion } & \multicolumn{1}{c}{ Wall motion } \\
\hline Initial event in the ischemic cascade & Follows induced perfusion abnormality \\
Does not require ischemia & Requires ischemia \\
Specific indicator of ischemia or ischemic potential & Nonspecific indicator of ischemia \\
Not significantly affected by loading conditions & Affected by loading conditions \\
Not significantly affected by resting wall motion abnormalities & Affected by resting wall motion abnormalities \\
Not generally affected by conduction abnormalities & Affected by conduction abnormalities \\
& \\
\hline Adapted from (3). & \\
\hline
\end{tabular}

the leading determinant of myocardial oxygen demand) increases flow demand, which secondarily increases flow. Pharmacologic stress, as delivered by dobutamine, acts similarly to increase flow indirectly through an increase in flow demands. However, dipyridamole and adenosine act directly to increase the coronary flow.

Indirect tests of the CFR, such as dynamic treadmill or bicycle exercise or dobutamine, seek to provoke ischemic perfusion and wall motion endpoints. Here, test sensitivity is influenced by, and directly related to, the ability of the intervention to augment demand. Direct tests of the CFR (e.g., dipyridamole or adenosine) are strong and generally maximal tests seeking to provoke flow heterogeneity and are best suited to the perfusion endpoint. Because they do not depend for their effect on the augmentation of coronary flow demands, they are less likely to be influenced by antianginal treatment such as $\beta$-blockers. Dipyridamole and adenosine, compared with dynamic exercise and dobutamine, test the CFR in a primary, direct manner, increasing coronary flow supply, not myocardial oxygen demand. Thus, dipyridamole and adenosine rarely produce ischemia, an imbalance in the coronary flow supply-to-demand ratio. Both direct and indirect tests of the CFR seek to maximize the hyperemic effect (Tables 1 and 2).

In those who can exercise sufficiently to increase the heart rate enough to maximally augment flow demands, both exercise and vasodilator stress may maximally test the CFR. Exercise is not only preferred clinically but also preferred to vasodilator stress in these types of patients because of its occasionally increased sensitivity (4). Exercise increases flow on the basis of an endothelium-dependent, flow-mediated coronary dilation of the distal coronary resistance vessels to supply myocardial oxygen requirements. Vasodilator stress agents, such as adenosine and dipyridamole, increase flow on the basis of a direct dilation of the coronary microcirculation independent of endothelial function. Because CAD brings about endothelial dysfunction, exercise may appropriately demonstrate this reduced CFR; the vasodilator agents may not. Verna et al. demonstrated this difference when they compared the results of exercise with vasodilator stress (4) in 36 selected patients. They found that exercise was not equal to pharmacologic stress, as the former yielded much larger stress-induced defects than did the latter.

Although vasodilator stress myocardial perfusion SPECT (MPS) has been found to have diagnostic and prognostic values similar to exercise, several additional reports of direct comparisons between exercise and vasodilator MPI have shown a greater extent, severity, and reversibility of defects with exercise, compared with the use of dipyridamole on the same subjects (5-9). Nonetheless, many patients who cannot exercise sufficiently to achieve the required threshold, for which exercise is insufficient to answer the clinical question, benefit from vasodilator stress testing.

\section{WHEN TO PERFORM PHARMACOLOGIC STRESS TESTING}

Pharmacologic stress testing is applied to evaluate the cause of symptoms, signs, or perceived risk from CAD in patients who cannot exercise or who cannot exercise sufficiently to perform an adequate diagnostic or prognostic

TABLE 2

Methods to Test CFR

\begin{tabular}{lc}
\hline Direct tests of CFR (vasodilator stress agents) & Indirect tests of CFR (exercise/dobutamine) \\
\hline $\begin{array}{ll}\text { Seek to provoke flow heterogeneity } & \text { Seek to provoke ischemia (perfusion or wall motion abnormality) } \\
\text { Best suited for perfusion endpoint } & \text { Fits either perfusion or function ischemic endpoint } \\
\text { (must be used with wall motion endpoint) }\end{array}$ \\
$\begin{array}{lc}\text { Less likely influenced by antianginal drugs } \\
\text { Strongest, most reproducible, tests of CFR }\end{array}$ & $\begin{array}{l}\text { Permit serial function analysis } \\
\text { Adapted from (3). }\end{array}$ \\
\hline
\end{tabular}


exercise test. It is said that safety should not be an issue, and patients should not be studied with pharmacologic stress if it is not safe for them to exercise. However, the patients who undergo pharmacologic stress are more debilitated and limited and are likely, as a group, to be at higher risk than those who exercise. Some patients, such as those studied early after myocardial infarction, may indeed be safely studied with pharmacologic but not with exercise stress.

The choice of the stress-testing method depends on the clinical question, or the test indication, not entirely on the patient's ability to exercise. For example, if an elderly woman experiences chest pain when pushing a shopping cart at a supermarket and we simply seek the cause of the pain, all that is required is an exercise test with an achieved workload similar to that of the activity that induced the symptom. Here, we would simply apply that stress, bring on the symptoms, monitor those symptoms, and observe. The specific heart rate and level of coronary flow demands achieved are not critical. However, if this same woman were to have high-risk vascular surgery, she would need a maximal assessment of coronary risk and CFR. If she could not exercise effectively or she was on treatment that blunted her rate-pressure product response, she would need effective pharmacologic stress testing in the form of coronary vasodilators.

Because these methods evaluate the perfusion endpoint, they must be performed with imaging. Vasodilator stress imaging with MPI currently accounts for roughly 50\% of all stress MPI.

\section{THE MECHANISM OF CORONARY VASODILATOR STRESS TESTING}

Figure 1 demonstrates the mechanism of action of adenosine on the coronary arteries. The agent causes dilation by interacting with an $\mathrm{A}_{2 \mathrm{~A}}$ receptor in what can be called a lowaffinity interaction. Here the agent is quickly released from the receptor, and its duration is short; its degradation by adenosine deaminase, a red-cell membrane-bound enzyme, is inhibited by dipyridamole, prolonging its vasodilator effect and increasing adenosine blood levels. In this way, the vasodilator properties of dipyridamole are based on increased levels of intrinsic adenosine, which binds to the $\mathrm{A}_{2 \mathrm{~A}}$ receptor (Fig. 1). It is well established that caffeine and other theophyllinelike drugs, such as aminophylline, inhibit the effects of adenosine and dipyridamole $(10,11)$. Aminophylline is the adenosine antidote, which preferentially binds the $A_{2 A}$ receptor. Generally used with dipyridamole to end the otherwise prolonged effects of the agent, aminophylline does not reduce dipyridamole levels or reduce elevated adenosine levels but rather displaces adenosine from the receptor, ending its effects. Because of its 12-s half-time, aminophylline is generally not used with short-lived adenosine stress, as adenosine effects generally end quickly after infusion cessation.

Although the mechanism is unclear, some work suggests that $\beta$-blockers can inhibit the effects of $A_{2 A}$ adenosine agonists, reducing test sensitivity but with some added prognostic value. Patients who have a reduced perfusion defect size on serial pharmacologic stress MPI with an added $\beta$-blocker appear to have a better prognosis and lower CAD risk than do those whose defects persist on MPI despite an added $\beta$-blocker (12). This result has been supported by data from a multicenter trial applying adenosine stress testing for prognosis in patients after myocardial infarction (MI) (13). The application of adenosine stress MPI soon after acute MI appears well able to identify a low-risk subgroup suitable for early hospital discharge (14). High-risk but stable survivors of MI may be treated

FIGURE 1. Shown are pathways of

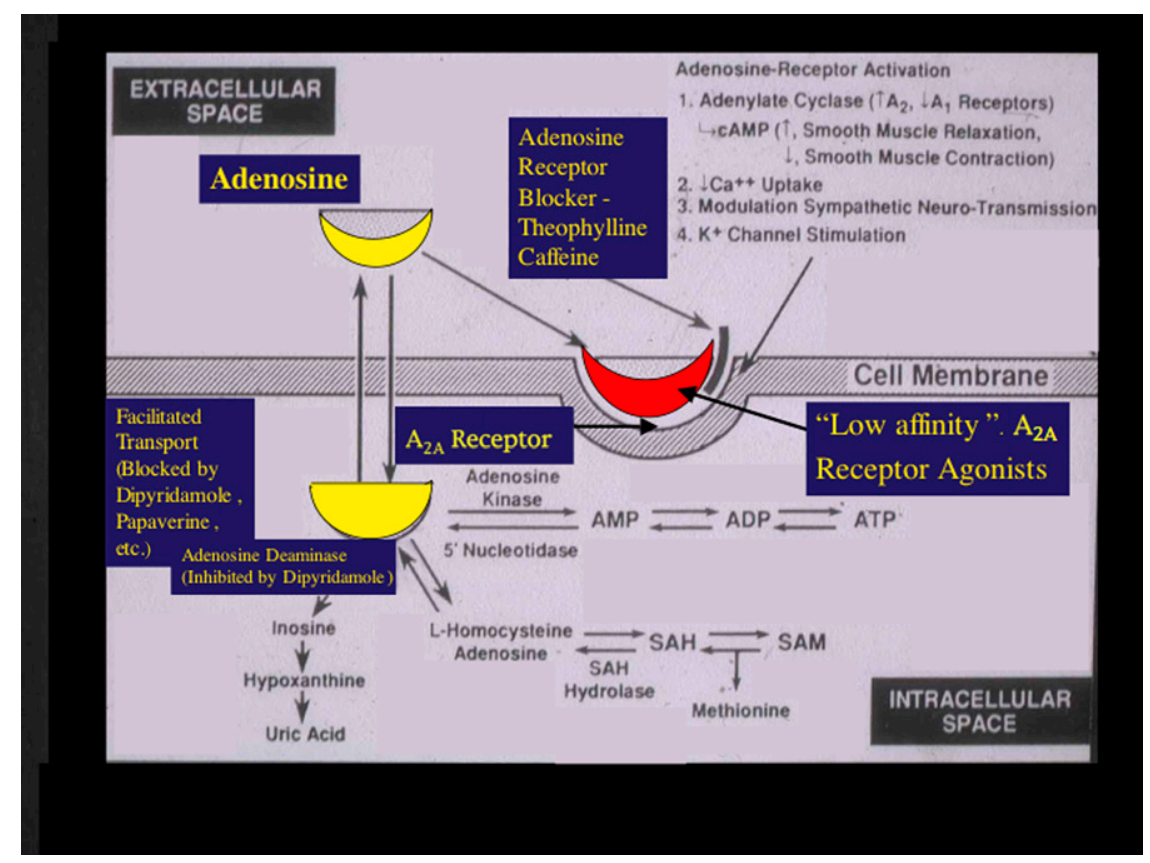
activation, and metabolism. (Adapted with permission of (10).) 
medically when they demonstrate a high degree of imagedefect improvement on repeated pharmacologic stress MPI (14), suggesting that the serial stress study indicates that the extent of myocardium at ischemic risk, and presumably the patient's coronary risk, are similarly reduced (15). Fewer myocardial perfusion abnormalities are seen during exercise than during adenosine stress in patients undergoing $\beta$-blocker therapy $(12,16)$. Adenosine stress testing should be preferred to exercise, to optimize diagnostic sensitivity in patients during $\beta$-blocker treatment.

Patients should not have caffeine for $24-48 \mathrm{~h}$ before testing and should be safely withdrawn from $\beta$-blocker treatment before the study, if possible. Dipyridamole, given therapeutically as an oral agent to reduce platelet adhesiveness in patients with prior strokes or as an ingredient of Aggrenox (Boehringer Ingelheim Pharmaceuticals, Inc.), may pose a danger during adenosine infusion, prolonging drug action and requiring an aminophylline antidote at the end of the test.

\section{NONSELECTIVE A $_{2 A}$ ADENOSINE RECEPTOR AGONISTS}

Adenosine and dipyridamole, the currently available vasodilators, are nonselective adenosine agonists that affect all subtypes of adenosine receptors. Adenosine may be infused in protocols requiring 4-6 min, whereas the dipyridamole infusion protocol takes 10-15 min. Despite their brevity, these agents frequently produce undesired side effects and infrequently produce complications (Table 3). Side effects are more frequent with adenosine than with dipyridamole, but rarely do they force premature test cessation. Although side effects are ameliorated, diagnostic accuracy is not advanced when low-level exercise is added to vasodilator stress testing (17). Induced systemic vasodilation often leads to headache, lightheadedness, flushing, nausea, reduced blood pressure, and increased heart rate. Although we all seek testing with patient comfort and would like to reduce side effects, of greatest concern are the potentially serious and even life-threatening complications. Nonspecific stimulation of the $\mathrm{A}_{2 \mathrm{~B}}$-adenosine receptors may lead to the complication of bronchospasm, and activation of the $\mathrm{A}_{1^{-}}$ adenosine receptors could result in bradycardia or heart block. However, patients with active bronchospasm, severely reduced 1 -s forced-expiratory volume $\left(\mathrm{FEV}_{1}\right)$, or evidence of advanced heart block are generally excluded from vasodilator stress testing with current agents. Such patients, if suitable, may be switched to exercise stress testing. Alterna-

TABLE 3

Physical, Chemical, and Clinical Characteristics of Current Pharmacologic Stress Agents

\begin{tabular}{|c|c|c|c|}
\hline \multirow[b]{2}{*}{ Characteristic } & \multicolumn{3}{|c|}{ Agent } \\
\hline & Dipyridamole & Adenosine & Dobutamine \\
\hline Source & Synthetic & Natural & Synthetic \\
\hline FDA approved for stress testing & Yes & Yes & No \\
\hline Mechanism & Tests CFR & Tests CFR & True ischemic stress \\
\hline Action on CFR & Indirect & Direct & Indirect \\
\hline Preparation & Simple & Simple & $\begin{array}{l}\text { Complicated (requires } \\
\text { trained nurse) }\end{array}$ \\
\hline Dosage & $\mathrm{mg} / \mathrm{kg}$ & $\mathrm{mg} / \mathrm{kg}$ & $\mathrm{mg} / \mathrm{kg}$ \\
\hline Infusion & $\begin{array}{l}\text { Timed pump (may be } \\
\text { hand titrated) }\end{array}$ & $\begin{array}{l}\text { Timed/isolated } \\
\text { intravenous source }\end{array}$ & $\begin{array}{l}\text { Pump titration } \\
\text { (requires trained nurse) }\end{array}$ \\
\hline Agent duration (half-life) & Prolonged ( $90 \mathrm{~min})$ & Very short (12 s) & Short (2.4 min) \\
\hline Intravenous line fails during infusion & Problematic & Redo & Problematic \\
\hline Variable infusion rate & Tolerable & Intolerable & Intolerable \\
\hline Stress-test duration & $\sim 10-12 \min$ & $\sim 4-8 \mathrm{~min}$ & 〜20-30 $\min$ \\
\hline Supervision and quality control & Modest & High & High \\
\hline Patient tolerance & High & High & Moderate \\
\hline Complications/side effects & Bronchospasm/heart block & Bronchospasm/heart block & $\begin{array}{l}\text { Ischemia/arrhythmia } \\
\text { hypotension }\end{array}$ \\
\hline Symptom duration & Usually brief & Brief & May be prolonged \\
\hline Safety & Like exercise test & Like exercise test & $\begin{array}{l}\text { Less than exercise } \\
\text { test (only apply to } \\
\text { selected patients) }\end{array}$ \\
\hline Safety with baseline dipyridamole & Preserved & Reduced & Preserved \\
\hline Antidote & Aminophylline & Discontinue & $\beta$-blocker \\
\hline Speed of reversal & Minutes & Seconds & Minutes \\
\hline Diagnostic indicator & Perfusion & Perfusion & Perfusion/wall motion \\
\hline Prognostic value & High & High & Modest \\
\hline Sensitivity with caffeine & Reduced & Reduced & Unchanged \\
\hline Sensitivity with $\beta$-blocker & Reduced? & Reduced? & Reduced \\
\hline Prognosis with $\beta$-blocker & Enhanced? & Enhanced? & Reduced \\
\hline dapted from (3). & & & \\
\hline
\end{tabular}




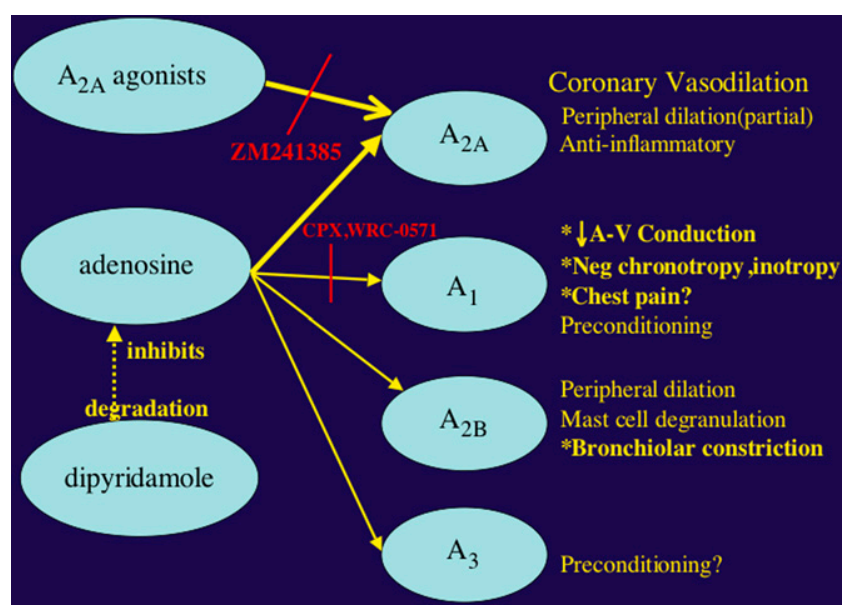

FIGURE 2. Four adenosine receptor subtypes- $-A_{1}, A_{2 A}, A_{2 B}$, and $A_{3}$-have been characterized and cloned. Stimulation of these receptors accounts for varied effects on electrical conduction, vasodilation, and bronchoconstriction. Illustrated are several adenosine receptor agonists and physiologic responses that result from stimulation of selective receptor subtypes. Shown also are inhibitors of 2 pathways. (Adapted with permission of (28).)

tively, patients with mild bronchospasm and an $\mathrm{FEV}_{1}$ above $50 \%$ of that predicted may be treated with bronchodilators and then studied by a conservative adenosine protocol. Here, safety may be increased with a serial monitored adenosine uptitration from a lower infusion rate to the test rate (18). Such efforts to gain application of vasodilator stress testing are often clinically important, because there may be no viable noninvasive alternative in exercise or dobutamine. Better would be the development of vasodilators more specifically targeting the coronary vasculature.

The direct coronary vasodilators adenosine and dipyridamole act directly on the coronary resistance vessels (the small arterioles and precapillaries) or through their inhibition of intrinsic adenosine degradation, to augment coronary flow and test the CFR (19) (Fig. 1; Tables 1 and 2). The method generally leads to abnormalities on MPI, simply with the production of a heterogeneous augmentation of coronary flow in the absence of induced ischemia $(20,21)$. In the absence of increasing myocardial oxygen demand, adenosine and dipyridamole uncommonly cause myocardial ischemia by a coronary-steal mechanism. Here, vasodilation and a related falling resistance in the normal bed result in a loss of the pressure gradient driving collateral flow, with withdrawal of collateral supply and resultant ischemia in severely stenotic, dependent beds. When a steal is induced, true ischemia is often accompanied by ischemic ST changes, a highly specific sign of ischemia in the setting of vasodilator stress (22). The prognostic value of vasodilator stress imaging has generally been shown to be equal to that of maximal and optimal exercise stress imaging.

Adenosine and dipyridamole-which are well able to induce abnormalities of the CFR, generally without inducing ischemia — are the most widely applied pharmacologic stress agents in the nuclear medicine laboratory.

\section{DOBUTAMINE STRESS}

In the absence of an ultrasound contrast agent that can directly monitor perfusion, adenosine and dipyridamole find little application to echocardiography, in which induced wall motion abnormalities are sought as the indicator of true coronary ischemia. Because of its ability to augment the determinants of myocardial oxygen demand and test the CFR, dobutamine is applied as an ischemic stress agent. Dobutamine stress MPI has been shown to be more sensitive for CAD diagnosis than is dobutamine stress echocardiography. The agent is applied widely for pharmacologic stress in the echocardiography laboratory but infrequently in the nuclear laboratory because of the ability of scintigraphic methods to monitor the hyperemic response and the related ability to apply the safer, more accurate vasodilator agents (23).

If dobutamine is ineffective in increasing myocardial oxygen demands, or if the patient develops intolerance early in the administration of dobutamine, its effect on the CFR is blunted. The ability of dobutamine to augment coronary flow and test the CFR is lower than that of adenosine, even when applied to maximal dose (24). Additionally, although sub-

TABLE 4

Chemical and Clinical Characteristics of New Selective $A_{2 A}$ Agonists

\begin{tabular}{|c|c|c|c|c|}
\hline \multirow[b]{2}{*}{ Characteristics } & \multicolumn{4}{|c|}{ Selective $A_{2 A}$ Agonists } \\
\hline & CGS21680 (X) & MRE-0470 Binodenoson & ATL-146e $(\mathrm{X})$ & CVT-3146 Regadenoson \\
\hline Selectivity & Low & High & Very high & Moderate \\
\hline Affinity & High & High & High & Moderate \\
\hline Potency & Moderate & High & Very high & Moderate \\
\hline Stable & Yes & Yes & Yes & Yes \\
\hline Onset & $1-2 \min$ & $1-2 \min$ & $1-2 \min$ & $<1 \min$ \\
\hline Duration & $>20 \min$ & $6-12 \min$ & $10-20 \mathrm{~min}$ & $3 \mathrm{~min}$ \\
\hline Trials & - & 2 (phase III) & 1 in 2003 & 2 \\
\hline FDA approved & - & To be submitted & - & YES \\
\hline
\end{tabular}

Adapted with permission of (28). 


\section{Regadenoson: moderate affinity and potency \\ Binodenoson: high affinity and potency \\ Functional selectivity: reduced activity for $A_{1}, A_{2 B}$, and $A_{3}$}
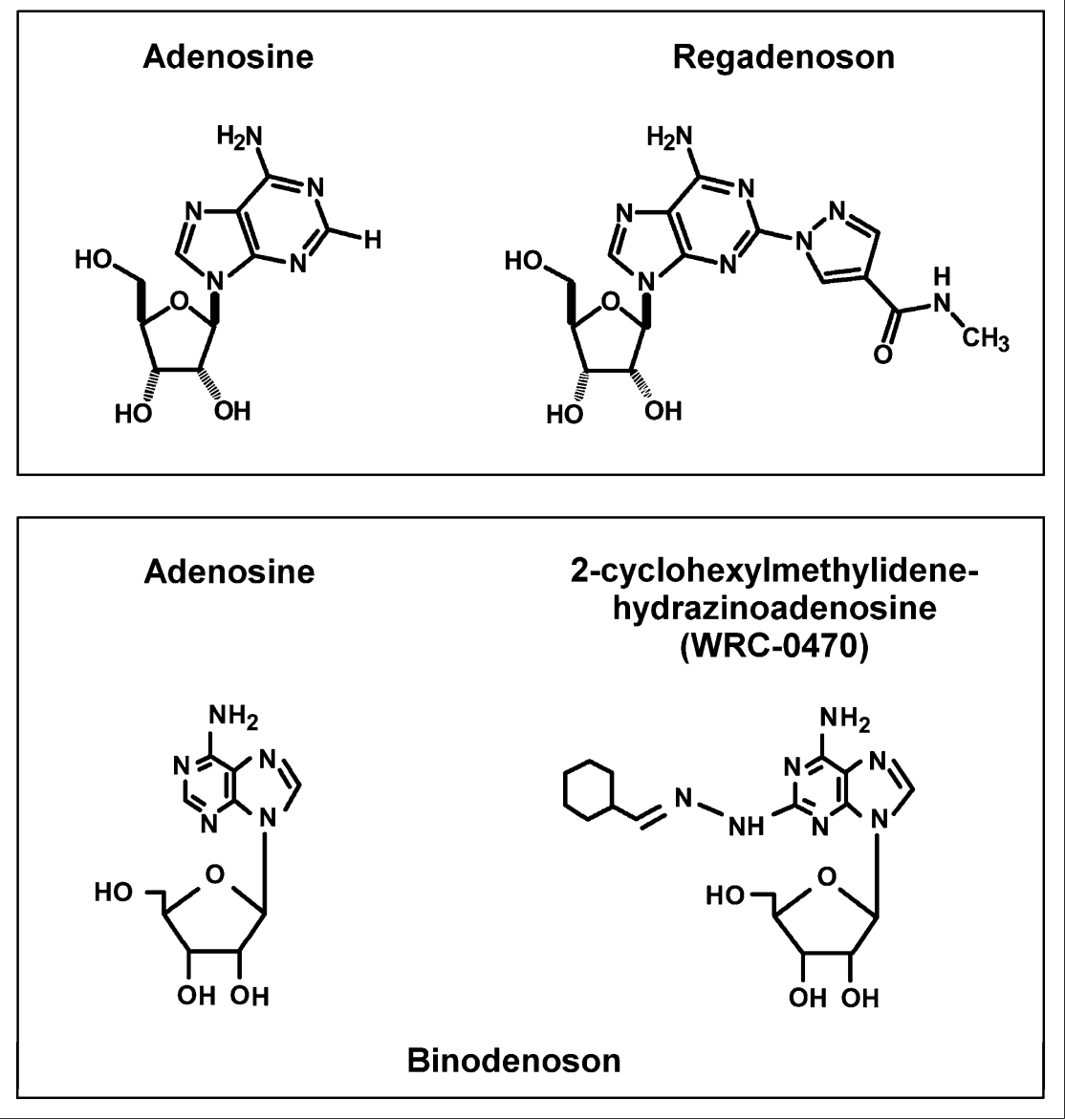

FIGURE 3. Shown are chemical compositions for regadenoson and binodenoson, compared with adenosine. Also presented are parameters of affinity and potency along with demonstration of its functional selectivity for $A_{2 A}$ receptors. Affinity relates to tightness of binding of agent to receptor and its resultant duration of action. Adenosine is a low-affinity agent that is quickly released. Higher affinity of new and more specific $A_{2 A}$ agonists does not seemingly interfere with preferential aminophylline binding and its use as antidote. jects are carefully selected with many exclusions, the complication rate is high (25), and not infrequently the effects of the agent force premature test cessation. The use of dobutamine is prohibited in the setting of acute MI, acute coronary syndromes, uncontrolled hypertension, aortic stenosis, dissecting aneurysms, and other conditions that are aggravated by the effects of the agent.

The 3-min stages of the incremental titration dobutamine stress protocol are modeled, for commercial reasons, after the 3-min stages of the standard exercise protocol. Its 2.4-min halftime indicates that the dobutamine dose would be better augmented at intervals of $10-15 \mathrm{~min}$ to permit the adequate buildup of drug levels and related effectiveness at each stage. However, commercially this interval would be unacceptable and too time-consuming for clinical application. Those who apply this 3-min interval must do so acknowledging the intrinsic loss of sensitivity early in the test and the potential dangers late in the test, when the earlier dose-related effects accumulate and manifest themselves unpredictably, where a higher dose is infused before the effects of earlier levels can occur. Although, in the absence of any alternative, dobutamine is applied widely by those who seek to use echocardiography as the stressimaging modality, the agent need only rarely and grudgingly be applied in the nuclear laboratory in selected patients without contraindications, when adequate exercise testing is not possible and when the risk of vasodilators is prohibitive.

\section{SELECTIVE A $A_{2 A}$ ADENOSINE RECEPTOR AGONISTS}

\section{The Issue}

The mechanism of adenosine-mediated vasodilation of human coronary arteries involves the activation of the adenosine cyclase signaling pathway via the adenosine receptor in the coronary endothelium (Fig. 1). However, several subtypes of adenosine receptors have been identified that are localized in tissues other than the coronary endothelium. Although the $\mathrm{A}_{2 \mathrm{~A}}$ adenosine receptors are localized on the surface of arterial vascular smooth muscle cells, the $A_{2 B}$ adenosine receptors predominate in the bronchial system and the $\mathrm{A}_{1}$-adenosine receptors concentrate in the atrioventricular node. Myocardial perfusion agents demonstrate the hyperemic effects of activating the $\mathrm{A}_{2 \mathrm{~A}}$ adenosine receptors 


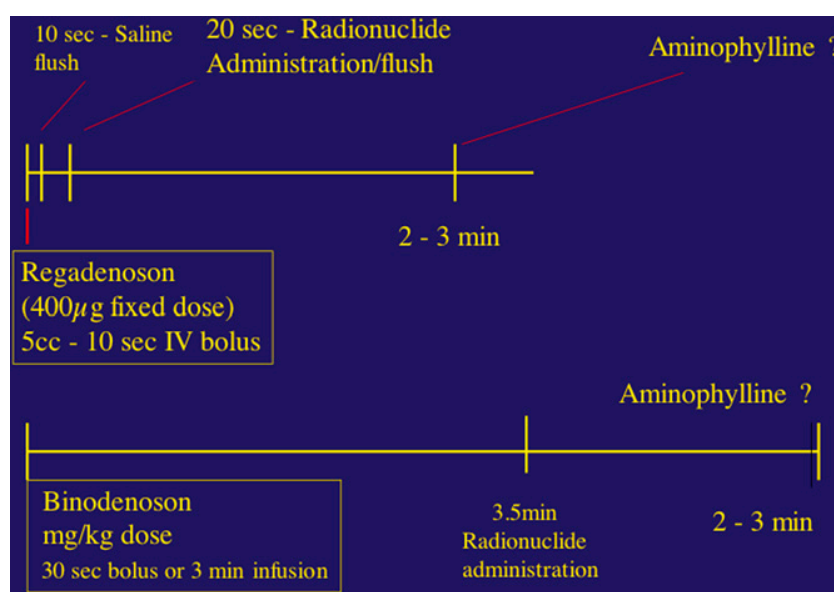

FIGURE 4. Shown diagrammatically are clinical infusion protocols recommended for regadenoson and binodenoson. These are designed on the basis of pharmacokinetics of the agents and their necessary interaction with the imaging agent.

in the coronary circulation, which specifically dilates the arterial vessels (26). The effects of a coronary vasodilator on $\mathrm{A}_{1}$ and $\mathrm{A}_{2 \mathrm{~B}}$ adenosine receptors are undesirable (Table 3).
In just such an effort to minimize these complications and side effects, several adenosine $\mathrm{A}_{2 \mathrm{~A}}$-specific receptor agonists have been developed, and results appear promising (Fig. 2) (27). One such agent is now Food and Drug Administration (FDA) approved, and another is in the late phase of clinical trials (2831). Increased heart rate may not always be the simple effect of vasodilation but may be the result of sympathetic stimulation (16) and so unaffected by selective receptor agonists.

\section{The Solution}

The ideal features of a vasodilator stress agent include the following: The agent should be a selective $\mathrm{A}_{2 \mathrm{~A}}$ agonist, with reduced side effects; there should be no AV block or bronchospasm, a minimal blood pressure and heart-rate response, and a rapid-effect onset and termination; a simplified delivery requiring no pump and bolus administration of a single dose for all patients would be desirable; and there should be an optimal level and duration of the hyperemic response, long enough to permit radiotracer extraction with short-lived and reduced side effects (27).

Table 4 shows the properties of agents that have long aspired to gain acceptance as $\mathrm{A}_{2 \mathrm{~A}}$ agonists. Two of those

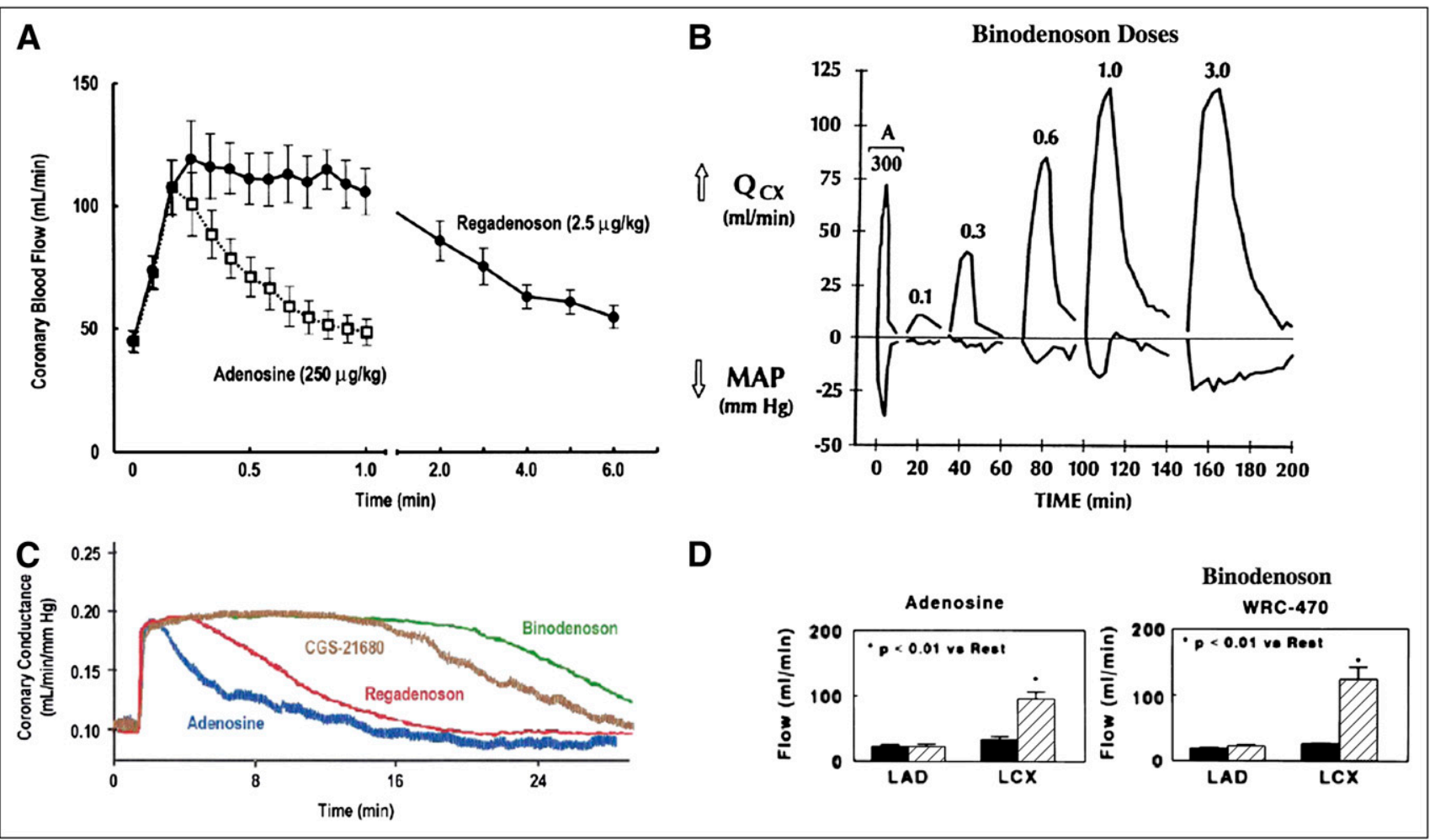

FIGURE 5. (A) Shown is time course of changes in coronary blood flow with regadenoson (solid curve) and adenosine (dashed curve). (Adapted with permission of (33).) (B) Shown above line for dogs are incremental changes in coronary flow ( $\left.Q_{C X}\right)$ noted with serial increases in binodenoson dosage, compared with adenosine dosage. Decremental changes in mean arterial pressure (MAP) are plotted below line. (Adapted with permission of (34).) (C) Shown is time course of changes in coronary conductance, coronary blood flow normalized for perfusion pressure, with regadenoson (red curve), binodenoson (green curve), adenosine (blue curve), and CGS-21680, an unsuccessful dilator that has been withdrawn. (Adapted with permission of (31).) (D) Shown is increased coronary flow with adenosine (left) and binodenoson, WRC-470 (right) in a dog with tight stenosis of left anterior descending (LAD) coronary artery but without evident left circumflex (LCX) disease. Flow at baseline is shown in black, and flow with respective dilators is shown with hatched bar. Note blunted LAD response; LCX responds fully, given presence of flow-limiting agent. The binodenoson seems to bring same or higher flow response. (Adapted with permission of (34).) 

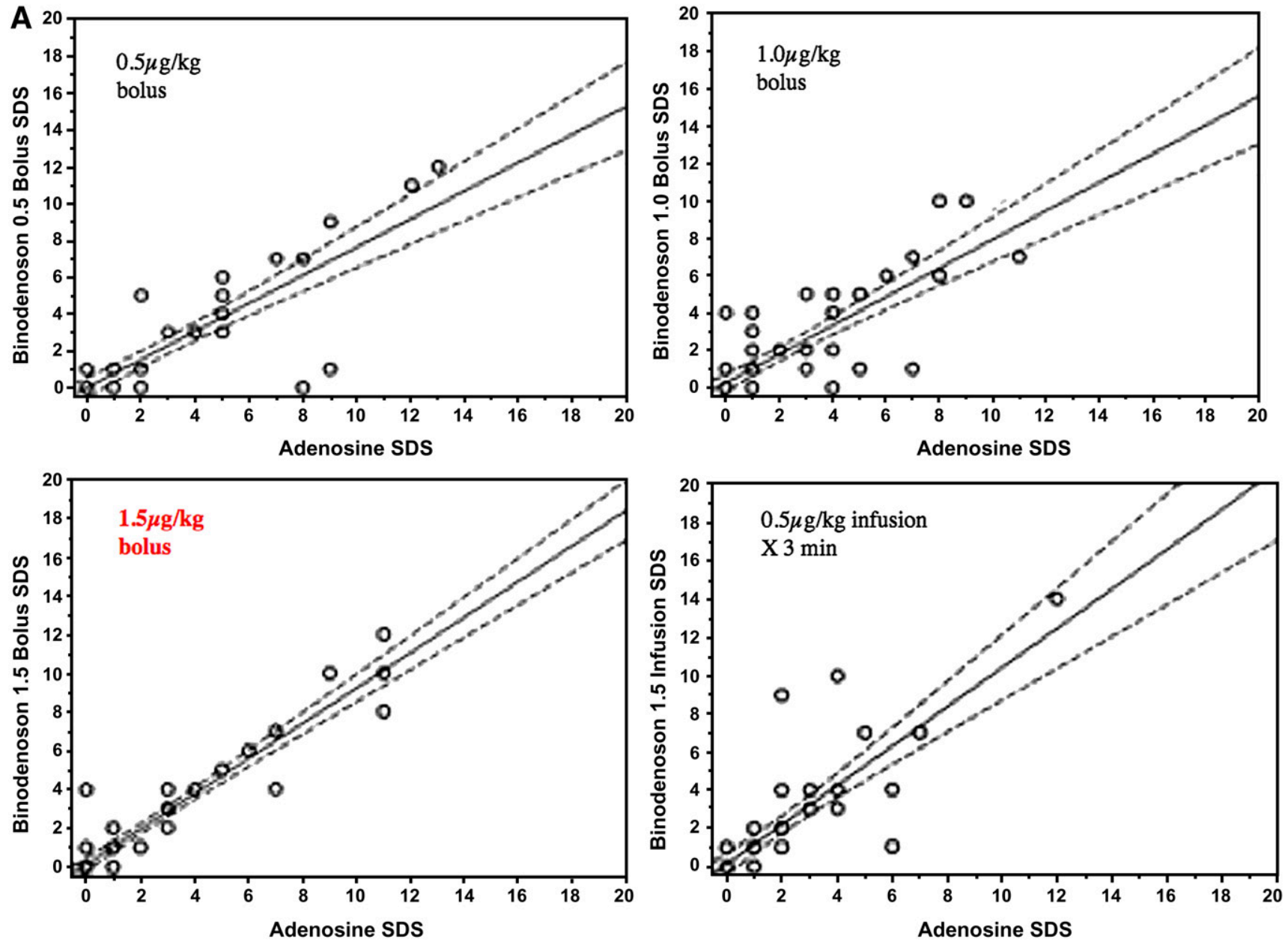

B

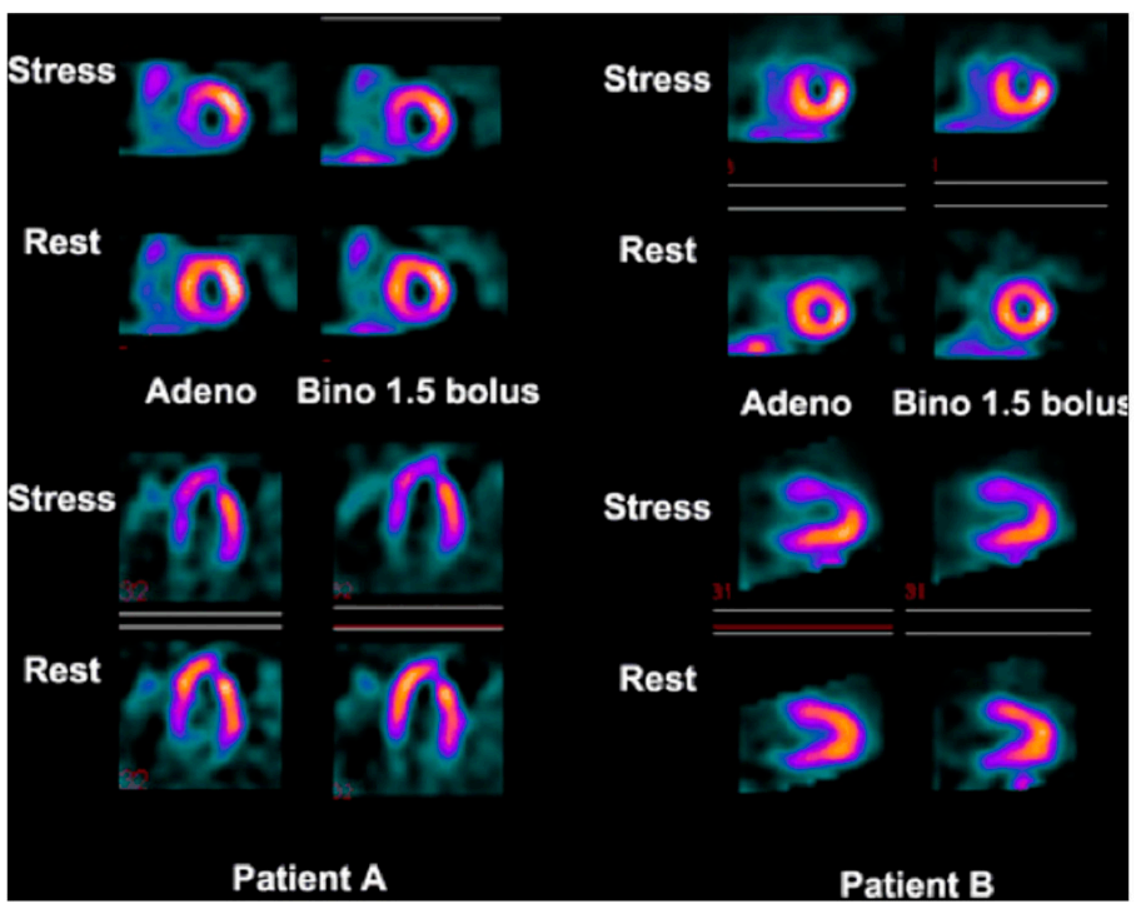

FIGURE 6. (A) Correlations with 4 dosing regimens. Summed defect scores (SDS) generated with adenosine correlated well with those using binodenoson in a $1.5 \mu \mathrm{g} / \mathrm{kg}$ bolus dosage. (B) Shown are rest and stress adenosine and binodenoson perfusion images in 2 case examples (patients A and B). Agreement is apparent. (Adapted with permission of (30).) 

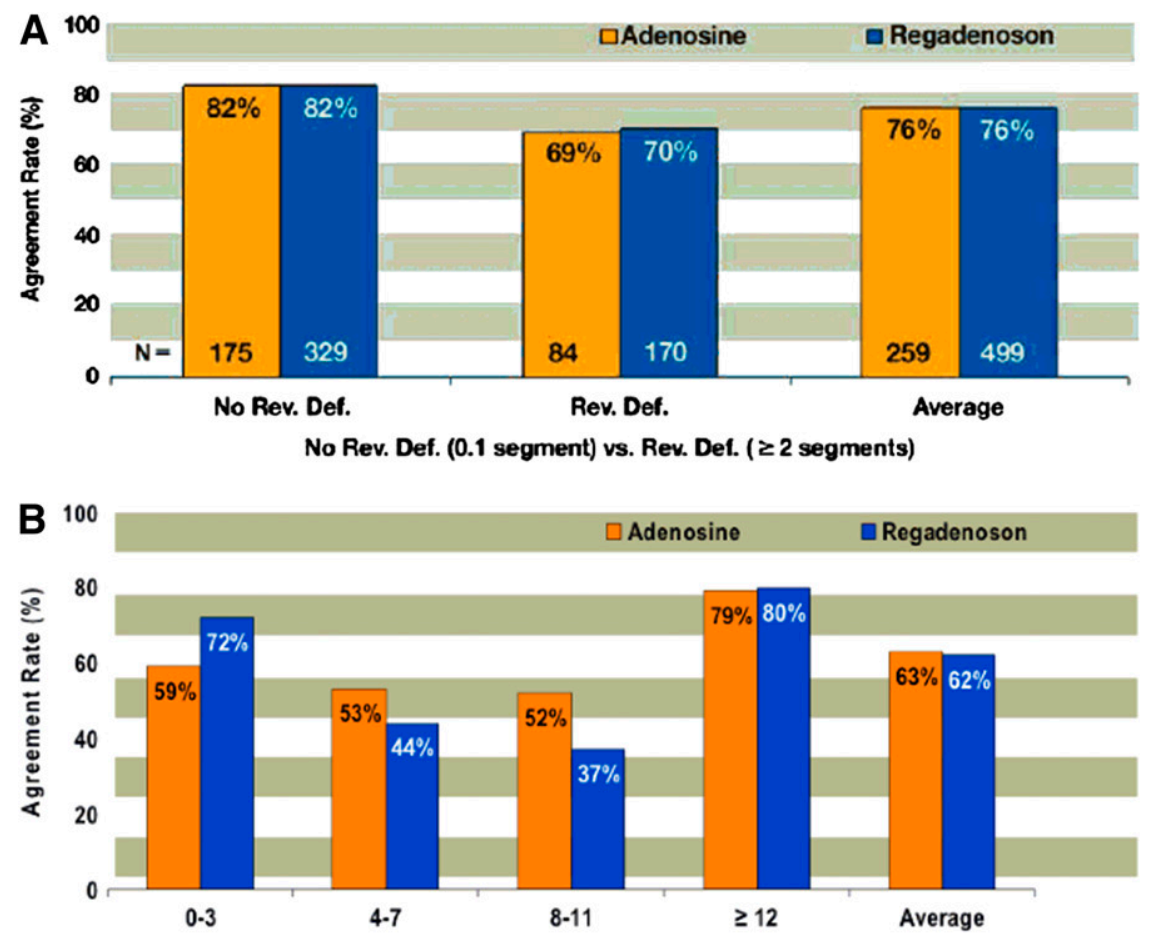

C

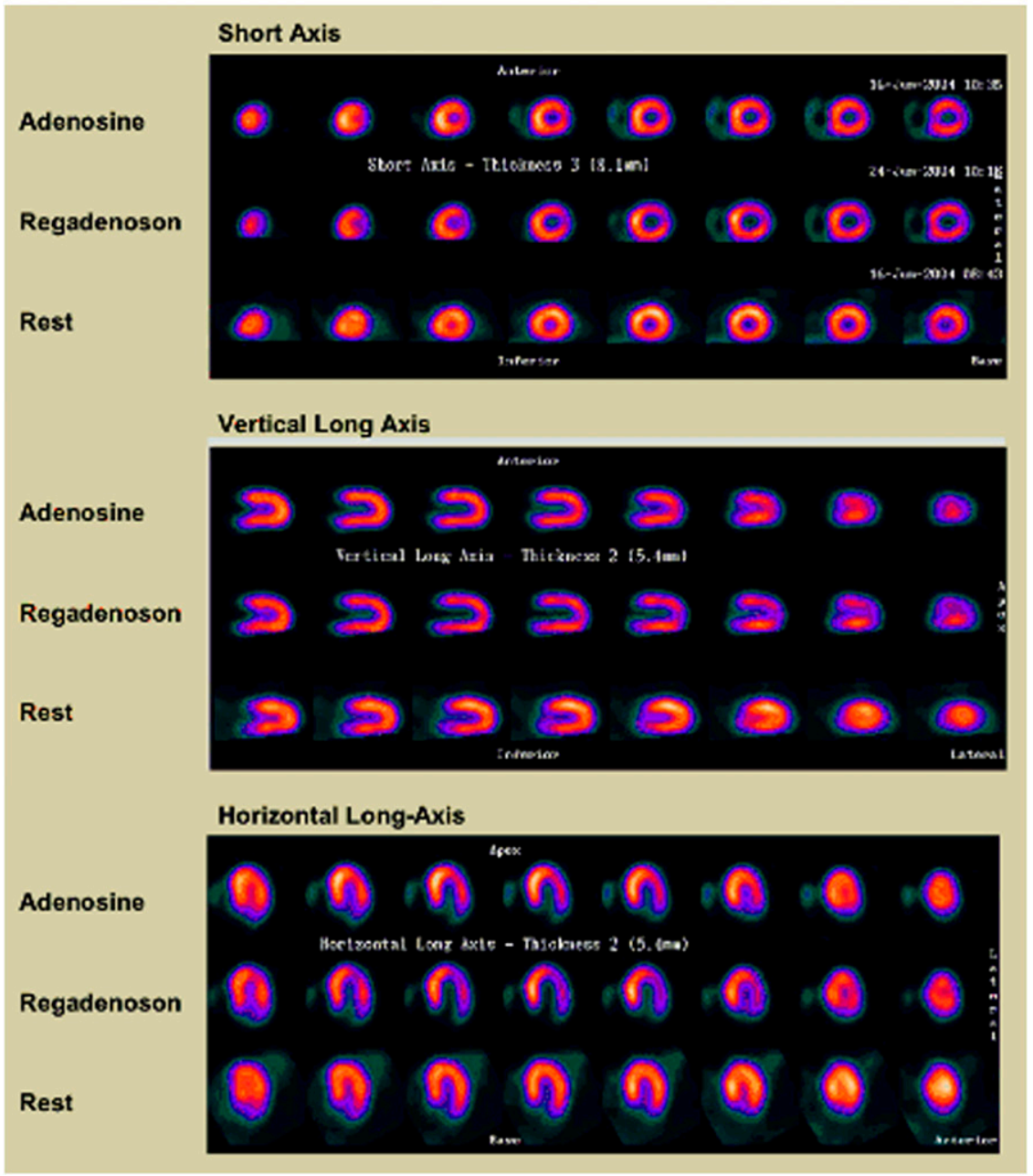

FIGURE 7. (A) Shown are agreement rates between adenosine-adenosine images (orange bars) and regadenosonadenosine images (blue bars) based on presence or absence of reversible defects. Equality between these comparisons is evident. (B) Shown are agreement rates between adenosine-adenosine images (orange bars) and regadenosonadenosine images (blue bars) by SSS, the summed stress score, based on a 17-segment model. Again, equality is evident. (C) Shown are SPECT images obtained with adenosine (top), with regadenoson (middle), and at rest (bottom) in 3 orthogonal views. Lateral reversible defect is seen on both sets of images and is more prominent with regadenoson. Rev. Def. = reversible defect. (Adapted with permission of (35).) 
shown have been marked with an $\mathrm{X}$ to indicate their failure, largely related to their long effect duration and other unfavorable characteristics. One agent, regadenoson, is already FDA approved and available for clinical testing as Lexiscan (Astellas Pharma US, Inc.). Although imperfect, these agents are significantly closer than current agents, and their success and adaptation will depend on their incremental clinical value, compared with current agents.

Figure 3 demonstrates the chemical formulae for adenosine, regadenoson, and binodenoson, with some of their physical characteristics (more completely presented in Table 4). The similarities of the 2 new synthetic agents to naturally occurring adenosine are clear. Regadenoson most resembles adenosine in its kinetics, with a similar time to onset and peak effects but with a prolonged duration (Table 4). Peak augmentation of CFR occurs more rapidly with regadenoson (seconds to minutes) than with binodenoson (several minutes), and the effect of regadenoson is briefer than that of binodenoson (5 vs. $20 \mathrm{~min}$, respectively) (Fig. 3; Table 4), influencing the duration and potentially the safety of the protocol and the potential need for the aminophylline antidote with persistent effects and side effects. Figure 4 demonstrates the clinical protocols for administration of regadenoson and binodenoson. Regadenoson has been formulated to be administered in a fixed-dose bolus, independent of patient weight. The administration of regadenoson, with its moderate receptor affinity, rapid onset, and short but seemingly adequate action duration, provides a swift, simple, and effective clinical protocol lasting 2-3 $\mathrm{min}$. The exact method of binodenoson administration is not yet established, with a protocol lasting about 5-7 min. Both fixed-dose and per-kilogram dosages have been tested. Both agents claim high $A_{2 A}$ receptor selectivity with little or no effect on $A_{1}$, $A_{2 B}$, or $A_{3}$ receptors $(31,32)$. Each has demonstrated in animal and human patient studies a high ability to dilate the coronary bed, with increased coronary blood flow, testing the CFR. Phase III patient studies with binodenoson are completed and have been submitted to the FDA.

In animals and human patients (Figs. 5A-5D), both agents have been shown to incrementally increase coronary flow with increasing dosage (Fig. 5B) up to 3 times, compared with baseline, with a variable effect duration (Fig. 5C). Given that regadenoson has been FDA approved in a fixed-dose protocol, this result is interesting and even unexpected. Ordinarily, such a dose would be optimal for a few patients, with many receiving relatively excess drug and being overdosed or receiving insufficient amounts of the agent and being underdosed. The effectiveness and safety of the single-dose regimen can be explained only by a high level of agent effectiveness and $\mathrm{A}_{2 \mathrm{~A}}$ receptor selectivity, or major side effects would be expected. Such specificity of receptor effect has been well demonstrated in animals (29-32). Like adenosine, regadenoson and binodenoson both modestly reduce arterial pressure in relation to dose (Fig. 5B). This augmentation of the CFR is blunted with a tight, flow-limiting stenosis (Fig. 5D). In studies conducted in patients with stress MPS, the size of defects induced generally parallel those seen in association with adenosine in patients with induced ischemia, with similar diagnostic accuracy of coronary disease.

Binodenoson augmentation of CFR has been shown to reach high levels in relation to several infusion rates, with the highest achieved level and the greatest duration in relation to a $1.5 \mu \mathrm{g} / \mathrm{kg} / \mathrm{min}$ dosage administered over $3 \mathrm{~min}$. Summed defect scores generated with adenosine correlated well with those using binodenoson in a $1.5 \mu \mathrm{g} / \mathrm{kg}$ bolus dose (Figs. 6A and 6B), with an effect duration of approximately 7-10 min. This duration of effect, although acceptable and potentially efficacious in a clinical protocol, is not as optimal as the 2- to 3-min duration of peak regadenoson effect, which better

TABLE 5

Adenosine and Regadenoson Symptoms

\begin{tabular}{lcrr}
\hline \multicolumn{1}{c}{ Symptom } & Adenosine $(n=267)$ & Regadenoson $(n=517)$ & $P$ (Fisher exact test) \\
\hline Any event & $210(79)$ & $409(79)$ & 0.93 \\
Any severe event & $18(7)$ & $25(5)$ & 0.32 \\
Flushing & $63(24)$ & $86(17)$ & 0.02 \\
Dyspnea & $49(18)$ & $128(25)$ & 0.05 \\
Headache & $42(16)$ & $148(29)$ & $<0.001$ \\
Chest discomfort & $42(16)$ & $57(11)$ & 0.07 \\
Chest pain & $34(13)$ & $41(8)$ & 0.04 \\
Angina pectoris & $22(8)$ & $40(8)$ & 0.78 \\
Feeling hot & $17(6)$ & $19(4)$ & 0.10 \\
Nausea & $12(4)$ & $29(6)$ & 0.61 \\
Dizziness & $9(3)$ & $35(7)$ & 0.05 \\
Abdominal discomfort & $5(2)$ & $32(6)$ & $<0.01$ \\
\end{tabular}

Data are presented as number of patients, with percentage in parentheses. Medical Dictionary for Regulatory Activities-preferred terms reported by $5 \%$ of patients or more in either treatment at any time after start of infusion are shown. Symptoms did not vanish with regadenoson and, in fact, differed little in their frequency, compared with those related to adenosine administration. This similarity may be related, in part, to the methods applied to determine the presence of symptoms. Adapted with permission of (35). 
TABLE 6

Adenosine and Regadenoson Tolerability

\begin{tabular}{|c|c|c|c|c|}
\hline Question & Tolerance level & Adenosine & Regadenoson & $\begin{array}{l}P \text { (Cochran-Mantel- } \\
\text { Haenszel test) }\end{array}$ \\
\hline How did you feel? & & & & $<0.001$ \\
\hline Comfortable & 1 & $85(32)$ & $200(39)$ & \\
\hline Slightly uncomfortable & 2 & $133(50)$ & $271(52)$ & \\
\hline Very uncomfortable & 3 & $38(14)$ & $39(8)$ & \\
\hline Extremely uncomfortable & 4 & $11(4)$ & $7(1)$ & \\
\hline Mean \pm SE & & $1.9 \pm 0.05$ & $1.7 \pm 0.03$ & \\
\hline $\begin{array}{l}\text { How did this test compare with } \\
\text { the first (open-label adenosine) test? }\end{array}$ & & & & $<0.001$ \\
\hline Much better & 1 & $41(15)$ & 177 (34) & \\
\hline Somewhat better & 2 & $74(28)$ & $129(24)$ & \\
\hline About the same & 3 & $108(40)$ & $126(24)$ & \\
\hline Somewhat worse & 4 & $30(11)$ & $74(14)$ & \\
\hline Much worse & 5 & $14(5)$ & $11(2)$ & \\
\hline Mean $\pm S E$ & & $2.6 \pm 0.06$ & $2.3 \pm 0.05$ & \\
\hline
\end{tabular}

Data are presented as number of patients, with percentage in parentheses, unless otherwise indicated. When symptoms were considered for their tolerability, regadenoson showed a clear advantage. Adapted with permission of (35).

matches the duration required for administration and extraction of the radiopharmaceutical during stress testing.

The ADVANCE phase 3 Multicenter Trial (35) compared the naturally occurring, nonselective vasodilator adenosine with the derived $\mathrm{A}_{2 \mathrm{~A}}$ receptor-specific agonist regadenoson. Adenosine stress MPS was performed in 787 patients who were then randomized to adenosine stress in 267 patients or regadenoson stress in 517 patients. The studies performed with the 2 agents and the studies performed twice with adenosine agreed for the number and size of induced image defects and their reversibility (Figs. 7A and 7B). A comparison of image findings in a typical case is shown in Figure 7C. Symptoms did not vanish with regadenoson. However, when the nature and frequency of symptoms induced with adenosine and regadenoson were compared, less flushing, dyspnea, headache, chest pain, dizziness, and abdominal discomfort with regadenoson were observed (Table 5). In addition, the degree of symptoms must have been extremely diminished, because regadenoson was much better tolerated (Table 6).

The induction of bronchospasm and the safety of regadenoson in patients with chronic obstructive pulmonary disease and asthma were investigated by Thomas et al. (36). In a masked manner, these authors measured respiratory flow rates and volumes in 49 patients with moderately $\left(\mathrm{FEV}_{1}=1.75 \mathrm{l}\right)$ or severely $\left(\mathrm{FEV}_{1}=1.0 \mathrm{l}\right)$ abnormal air movement with regadenoson and compared findings with those in a placebo group without imaging. Patients on oxygen or steroids or with wheezing before testing were excluded. No significant difference in reduction of $\mathrm{FEV}_{1}$ or new onset of wheezing between regadenoson and placebo was observed. No patient needed treatment with bronchodilators or oxygen. Leaker et al. (37) conducted a randomized, double-blind, placebo-controlled crossover trial of the effects of regadenoson on airway resistance and $\mathrm{FEV}_{1}$ in patients with asthma with a positive adenosine monophosphate challenge test. In all cases except
1, the measured ratio of $\mathrm{FEV}_{1}$ with regadenoson to baseline $\mathrm{FEV}_{1}$ was significantly increased. $\mathrm{FEV}_{1}$ in all patients returned to baseline after drug termination, without aminophylline.

Although the agent is FDA approved and will soon be available everywhere, there is much about regadenoson that we do not know as it approaches clinical application. What is not known about the regadenoson includes the use of the agent in patients with bronchospasm, the incidence of $\mathrm{AV}$ block, the effect of caffeine, the effect of $\beta$-blockers, the meaning of a test with a blunted hemodynamic and symptomatic response, the value of added exercise, the value of transient ischemic dilation, the effects of left bundle branch block, the ability to appreciate related ischemia, the frequency of related ischemia, the safety of the agent with renal insufficiency, the applicability of the agent to stress testing with PET and MRI, and the effects of the singledose protocol (is it related to overdosing or underdosing?).

\section{A SPECULATION}

Dipyridamole works slowly and by an indirect mechanism. Infusing this agent in 1 min rather than 4 min, or even bolusing, results in no discernible toxicity but only a question as to the timing of its peak effect and of the radionuclide injection. Adenosine acts rapidly and directly and cannot be bolused without a clear toxic effect. Regadenoson acts rapidly and directly but can be given safely as a single-dose bolus to patients of all weights. Regadenoson avoids toxicity only if its extracoronary effects are blunted, suggesting that it is likely a more specific $\mathrm{A}_{2 \mathrm{~A}}$ agonist than we know.

\section{REFERENCES}

1. Gould KL, Libscomb K, Hamilton GW. Physiologic basis for assessing critical coronary stenosis: instantaneous flow response and regional distribution during coronary hyperemia as measures of coronary flow reserve. Am J Cardiol. 1974; 33:87-95 
2. Sigwart U, Grbic M, Payot M. Ischemic events during coronary artery balloon obstruction. In: Rutishauser W, Roskamm H, eds. Silent Myocardial Ischemia. Berlin, Germany: Springer-Verlag; 1984:29-36.

3. Botvinick EH. Stress imaging: current clinical options for the diagnosis, localization and evaluation of coronary artery disease. Med Clin N Amer. 1995;79:1025-1061.

4. Verna E, Ceriani L, Provasoli S, Scotti S, Ghiringhelli S. Larger perfusion defects with exercise compared with dipyridamole SPECT (exercise-dipyridamole mismatch) may reflect differences in epicardial and microvascular coronary dysfunction: when the stressor matters. J Nucl Cardiol. 2007;14:818-826.

5. Lee J, Chae SC, Lee K, Heo J, Iskandrian AS. Biokinetics of thallium-201 in normal subjects: comparison between adenosine, dipyridamole, dobutamine and exercise. J Nucl Med. 1994;35:535-541.

6. Levine MG, Ahlberg AW, Mann A, et al. Comparison of exercise, dipyridamole, adenosine, and dobutamine stress with the use of Tc-99m tetrofosmin tomographic imaging. J Nucl Cardiol. 1999;6:389-396.

7. Chow BJ, Beanlands RS, Lee A, et al. Treadmill exercise produces larger perfusion defects than dipyridamole stress N-13 ammonia positron emission tomography. J Am Coll Cardiol. 2006;47:411-416.

8. Kugiyama K, Ohgushi M, Motoyama T, et al. Nitric oxide-mediated flowdependent dilation is impaired in coronary arteries in patients with coronary spastic angina. J Am Coll Cardiol. 1997;30:920-926.

9. David N, Marie PY, Angioi M, et al. Dipyridamole and exercise SPET provide different estimates of myocardial ischaemic areas: role of the severity of coronary stenoses and of the increase in heart rate during exercise. Eur J Nucl Med. 2000;27:788-799.

10. Verani MS. Adenosine thallium-201 myocardial perfusion scintigraphy. Am Heart J. 1991;122:269-278.

11. Lapeyre AC III, Goraya TY, Johnston DL, Gibbons RJ. The impact of caffeine on vasodilator stress perfusion studies. J Nucl Cardiol. 2004;11:506-511.

12. Müller-Suur R, Eriksson SV, Strandberg LE, Mesko L. Comparison of adenosine and exercise stress test for quantitative perfusion imaging in patients on betablocker therapy. Cardiology. 2001;95:112-118.

13. Mahmarian JJ, Shaw LJ, Olszewski GH, Pounds BK, Frias ME, Pratt CM. Adenosine sestamibi SPECT post-infarction evaluation (INSPIRE) trial: a randomized, prospective multicenter trial evaluating the role of adenosine Tc$99 \mathrm{~m}$ sestamibi SPECT for assessing risk and therapeutic outcomes in survivors of acute myocardial infarction. J Nucl Cardiol. 2004;11:458-469.

14. Mahmarian JJ, Shaw LJ, Filpchuk NG, et al. for the INSPIRE Investigators. A multinational study to establish the value of early adenosine technetium-99m sestamibi myocardial perfusion imaging in identifying a low risk group for early hospital discharge after acute myocardial infarction. J Am Coll Cardiol. 2006;48:2448-2457.

15. Mahmarian JJ, Dakik HA, Filpchuk NG, et al. for the INSPIRE Investigators. An initial strategy of intensive medical therapy is comparable to that of coronary revascularization for suppression of scintigraphic ischemia in high-risk but stable survivors of acute myocardial infarction. J Am Coll Cardiol. 2006;48:2458-2470.

16. Dhalla AK, Wong MY, Wang L, Belardinelli CV. Mechanism of tachycardia during myocardial perfusion imaging with adenosine [abstract]. J Nucl Cardiol. 2004;11(suppl):S2.

17. Holly TA, Satran A, Bromet DS, et al. The impact of adjunctive adenosine infusion during exercise myocardial perfusion imaging: results of the Both Exercise and Adenosine Stress Test (BEAST) trial. J Nucl Cardiol. 2003;10:291-296.

18. Henzlova MJ, Cerqueira MD, Mahmarian JJ, Yao SS, Quality Assurance Committee of the American Society of Nuclear Cardiology. Stress protocols and tracers. J Nucl Cardiol. 2006;13:e80-e90.
19. Klocke FJ. Coronary blood flow in man. Prog Cardiovasc Dis. 1976;19: $117-166$.

20. Hoffman JIE, Baer RW, Hanley FL, et al. Regulation of transmural myocardial blood flow. J Biomech Eng. 1985;107:2-9.

21. Kern MJ. Walking with Sir William: reflections on collateral steal, recruitment, and ischemic protection. J Am Coll Cardiol. 2006;48:66-69.

22. Cosmai EM, Heller GV. The clinical importance of electrocardiographic changes during pharmacologic stress testing with radionuclide myocardial perfusion imaging. J Nucl Cardiol. 2005;12:466-472.

23. Martin TW, Seaworth JF, Johns JP, et al. Comparison of adenosine, dipyridamole and dobutamine in stress echocardiography. Ann Intern Med. 1992;116:190-196.

24. Jagathesen R, Barnes E, Rosen SD, Foale RA, Camici PG. Comparison of myocardial blood flow and coronary flow reserve during dobutamine and adenosine stress: implications for pharmacologic stress testing in coronary artery disease. J Nucl Cardiol. 2006;13:324-332.

25. Lattanzi F, Picano E, Adamo E, Varga A. Dobutamine stress echocardiography: safety in diagnosing coronary artery disease. Drug Saf. 2000;22:251-262.

26. Sato A, Terata K, Miura H, et al. Mechanism of vasodilation to adenosine in coronary arterioles from patients with heart disease. Am J Physiol Heart Circ Physiol. 2005;288:H1633-H1640.

27. Cerqueira MD. The future of pharmacologic stress: selective $A_{2 A}$ adenosine receptor agonists. Am J Cardiol. 2004;94:33D-40D.

28. Hendel RC, Jamil T, Glover DK. Pharmacologic stress testing: new methods and new agents. J Nucl Cardiol. 2003;10:197-204.

29. Bateman TM, Cerqueira MD, Iskandrian AE, et al. Initial clinical experience with regadenoson, a novel selective $\mathrm{A}_{2 \mathrm{a}}$ agonist for pharmacologic stress singlephoton emission computed tomography myocardial perfusion imaging. $J \mathrm{Am}$ Coll Cardiol. 2005;46:2069-2075.

30. Udelson JE, Heller GV, Wackers FJ, et al. Randomized, controlled dose-ranging study of the selective adenosine $\mathrm{A} 2 \mathrm{~A}_{\mathrm{A}}$ receptor agonist binodenoson for pharmacological stress as an adjunct to myocardial perfusion imaging. Circulation. 2004;109:457-464.

31. Gao Z, Li Z, Baker SP, et al. Novel short-acting $\mathrm{A}_{2 \mathrm{~A}}$ adenosine receptor agonists for coronary vasodilation: inverse relationship between affinity and duration of action of $\mathrm{A}_{2 \mathrm{~A}}$ agonists. J Pharmacol Exp Ther. 2001;298:209-218.

32. Zablocki J, Palle V, Blackburn B, et al. 2-substituted pi system derivatives of adenosine that are coronary vasodilators acting via the $\mathrm{A} 2 \mathrm{~A}_{\mathrm{A}}$ adenosine receptor. Nucleosides Nucleotides Nucleic Acids. 2001;20:343-360.

33. Zhao G, Linke A, Xu X, et al. Comparative profile of vasodilation by CVT-3146: a novel A2A receptor agonist, and adenosine in conscious dogs. J Pharmacol Exp Ther. 2003;307:182-189.

34. Glover DK, Ruiz M, Yang JY, et al. Pharmacologic stress thallium scintigraphy with 2-cyclohexylmethylidenehydrazinoadenosine (WRC-0470): a novel, shortacting adenosine A2A receptor agonist. Circulation. 1996;94:1726-1732.

35. Iskandrian AE, Bateman TM, Belardinelli L, et al. for the ADVANCE MPI Investigators. Adenosine versus regadenoson comparative evaluation in myocardial perfusion imaging: results of the ADVANCE phase 3 multicenter international trial. J Nucl Cardiol. 2007;14:645-658.

36. Thomas GS, Tammelin BR, Schiffman GL, et al. Safety of regadenoson, a selective adenosine $\mathrm{A}_{2 \mathrm{~A}}$ agonist, in patients with chronic obstructive pulmonary disease: a randomized, double-blind, placebo-controlled trial (RegCOPD trial). J Nucl Cardiol. 2008;15:319-328.

37. Leaker BR, O'Connor B, Hansel TT, et al. Safety of regadenoson, an adenosine $\mathrm{A}_{2 \mathrm{~A}}$ receptor agonist for myocardial perfusion imaging, in mild asthma and moderate asthma patients: a randomized, double-blind, placebo-controlled trial. J Nucl Cardiol. 2008;15:329-336. 